\title{
Depth resolution enhancement in optical scanning holography with a dual-wavelength laser source
}

\author{
Jun Ke, ${ }^{1}$ Ting-Chung Poon, ${ }^{2}$ and Edmund Y. Lam ${ }^{1, *}$ \\ ${ }^{1}$ Imaging Systems Laboratory, Department of Electrical and Electronic Engineering, \\ University of Hong Kong, Pokfulam, Hong Kong \\ ${ }^{2}$ Bradley Department of Electrical and Computer Engineering, Virginia Tech, Blacksburg, Virginia, USA \\ ${ }^{*}$ Corresponding author: elam@eee.hku.hk
}

Received 5 August 2011; revised 15 September 2011; accepted 5 October 2011; posted 8 November 2011 (Doc. ID 152468); published 5 December 2011

\begin{abstract}
In this paper, we use two point sources to analyze the depth resolution of an optical scanning holography $(\mathrm{OSH})$ system with a single-wavelength source. A dual-wavelength source is then employed to improve it, where this dual-wavelength OSH (DW-OSH) system is modeled with a linear system of equations. Object sectioning in DW-OSH is obtained with the Fourier domain conjugate gradient method. Simulation results show that, with the two source wavelengths at $543 \mathrm{~nm}$ and $633 \mathrm{~nm}$, a depth resolution at $2.5 \mu \mathrm{m}$ can be achieved. Furthermore, an OSH system emulator is provided to demonstrate the performance of DW-OSH compared with a conventional OSH system. (C) 2011 Optical Society of America

OCIS codes: $\quad 090.1995,100.3190,100.3020,110.1758$.
\end{abstract}

\section{Introduction}

Optical scanning holography (OSH) [1] is a digital holographic $(\mathrm{DH})$ imaging technique that captures the three-dimensional (3D) information of an object with a single two-dimensional (2D) scanning process. In an OSH system, two pupil functions are used with an optical heterodyning technique to generate two illumination source beams. After transmitting through an object, the interference of the two beams is detected by a single detector with a $2 \mathrm{D}$ rasterscanning mechanism, resulting in a complex hologram after some processing of the raw measurements. To date, several applications of OSH have been demonstrated, with 3D microscopy being the most mature. The advantages of OSH over conventional microscopy, such as the ability to control the degree of coherence of the imaging process by the size of a photodetector and the ability to use large nonimaging detectors for weakly fluorescent samples, have been reported over the last decade $[\underline{2}, \underline{3}]$.

0003-6935/11/34H285-12 $\$ 15.00 / 0$

(C) 2011 Optical Society of America
In DH microscopy, an important step is to obtain an individual object section from a 3D object, a process known as sectioning or sectional image reconstruction. The conventional method for sectioning in OSH uses the conjugate of the system impulse response, but the main drawback is that a large residue signal from the undesired object section remains, which is often called the defocus noise. To improve this, methods using Wiener filter [4], Wigner distribution [5], and inverse imaging [6,7] have been develope $\bar{d}$. Meanwhile, other optical techniques to improve the OSH system performance have also been proposed. For instance, instead of using a plane wave and a spherical wave to generate the impulse response, one can use a positive and a negative curvature spherical source beams for object illumination [2]. In doing so, the lateral resolution is doubled. Another technique is to use a random-phase pupil to transform the defocus sectional images into "speckle-like noise," then by averaging over multiple sectioning results one can reconstruct individual sections while suppressing the out-of-focus haze [8]. These two methods can be categorized as a point spread function (PSF) engineering approach to improve the resolution in a conventional OSH system. 
In this paper, we present another strategy to manipulate the OSH system impulse response for depth resolution improvement, using a dual-wavelength source. Traditionally with a single wavelength, the source beam propagates through the two pupils for object illumination. The complex hologram can be considered an imaging result of the object using a set of complex Fresnel zone plates (FZPs). The axial or depth resolution of an FZP is high if the numerical aperture (NA) used in the scanning system is large, where the NA is determined by multiple OSH system parameters such as the source wavelength. Here, we modify the system's source wavelength and collect two different sets of measurements. With proper image reconstruction schemes, we are able to combine the information from the two measurements to achieve better depth resolution.

The paper is organized as follows. In Section 2, the depth resolution in a conventional OSH system, which we will refer to as single-wavelength $\mathrm{OSH}$ (SW-OSH) here, is analyzed. Then in Section 3 , a dual-wavelength OSH (DW-OSH) system is described in detail. To reconstruct sectional images from the complex hologram, we use a Fourier domain conjugate gradient (CG) method, which is also discussed in this section. Simulation results are presented in Section 4 to demonstrate the improvement of DW-OSH over SW-OSH in terms of depth resolution. Then an OSH system simulator is described in Section 5 that can be of use in further study on OSH, followed by some concluding remarks given in Section $\underline{6}$.

\section{OSH Depth Resolution Analysis}

Depth resolution is a critical parameter in 3D imaging. In this section, an object of two point sources located at two axial planes is used to analyze the depth resolution of an OSH system. We begin with some background about its imaging principles.

\section{A. OSH System with a Single-Wavelength Source}

An OSH system diagram is shown in Fig. 1. In this on-axis DH imaging system, the laser source is split into two parts by a beam splitter BS1. Each part is used to generate an object illumination beam, either with a plane wavefront or with a spherical wavefront. For the latter, one of the two pupil functions, $p_{1}(x, y)$, is set to equal unity, which is physically a fully open mask. The other pupil function, $p_{2}(x, y)$, is set to be a delta function (physically a pinhole) for the plane wavefront [9]. The combination of these two waterfronts through beamsplitter BS2 is used to scan the object in 2D. A single detector is then used to detect the scattered wavefront after the object as lens 3 is used to collect all the scattered light from the object.

Note that this only preserves the wavefront amplitude information. To capture the phase, optical heterodyning is implemented in OSH [1]. Thus, the temporal frequency of one source beam is shifted by an acousto-optic frequency shifter (AOFS) from $\omega_{0}$ to $\omega_{0}+\Omega$. With this device, the detector measurements record not only the interference pattern of the two beams with different wavefronts, but also the phase of the interference signal through heterodyning of the two beams with different temporal frequencies. Using an electronic demodulator to process the measurements, we can recover the complex scattered wavefront from the object. In what follows we use the term "OSH system measurement" to refer to the demodulated complex signal from the detector raw measurements. We assume to have $N$ data points altogether.

The OSH system has the PSF given by [1]

$$
h(x, y ; z)=-j \frac{k}{2 \pi z} \exp \left\{j \frac{k}{2 z}\left(x^{2}+y^{2}\right)\right\},
$$

where $x, y, z$ are the coordinates and $k$ is the light source wavenumber. The corresponding optical transfer function (OTF) is

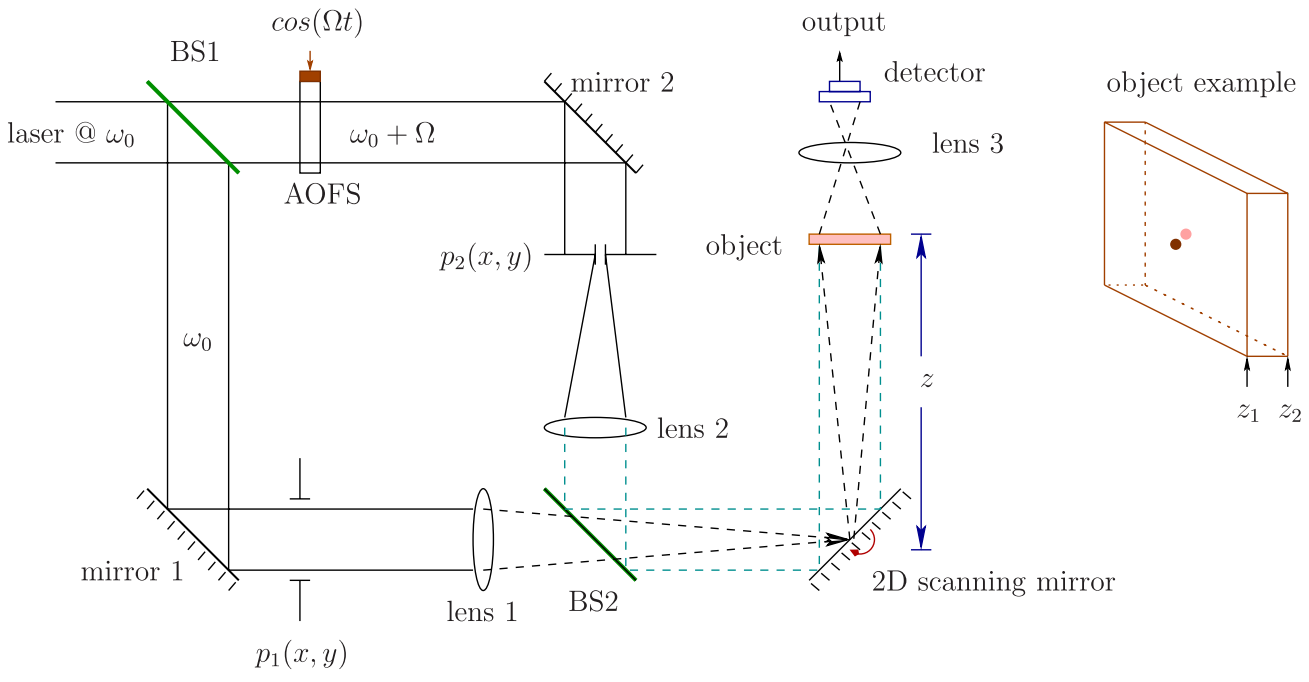

Fig. 1. (Color online) An OSH system diagram. 


$$
H\left(k_{x}, k_{y} ; z\right)=\mathcal{F}\{h(x, y ; z)\}=\exp \left\{-j \frac{z}{2 k}\left(k_{x}^{2}+k_{y}^{2}\right)\right\}
$$

Different from other DH systems, the OSH system as shown in Fig. 1 is used to image the intensity of a 3D object. Therefore, the OSH system measurements only contain the object amplitude information. For a $3 \mathrm{D}$ object represented by a complex field $O(x, y ; z)$, the OSH system measurements can be written as

$$
g(x, y)=\int_{-\infty}^{\infty}|O(x, y ; z)|^{2} * h(x, y ; z) \mathrm{d} z,
$$

where " $*$ " indicates a $2 \mathrm{D}$ convolution in the $(x, y)$ plane [6]. To simplify the notation, we let $f(x, y ; z)=$ $|O(x, y ; \bar{z})|^{2}$, which would be a nonnegative function. We therefore rewrite the system measurement as

$$
g(x, y)=\int_{-\infty}^{\infty} f(x, y ; z) * h(x, y ; z) \mathrm{d} z .
$$

When we want to reconstruct the object information at a depth of $z_{0}$ from the hologram, the conventional method is to convolve $g(x, y)$ with the conjugate of the system PSF at $z_{0}$, i.e., $h^{*}\left(x, y ; z_{0}\right)$ [1]. In Fourier domain, this method can be written as

$$
\mathcal{F}\left\{f_{\text {est }}\left(x, y ; z_{0}\right)\right\}=\mathcal{F}\{g(x, y)\} H^{*}\left(k_{x}, k_{y} ; z_{0}\right),
$$

and that $H^{*}\left(k_{x}, k_{y} ; z_{0}\right)=\exp \left\{j \frac{z_{0}}{2 k}\left(k_{x}^{2}+k_{y}^{2}\right)\right\}$. As discussed in the introduction, other methods for sectional image reconstruction have been developed, which we will discuss in more details later.

\section{B. Depth Resolution Analysis with Two Point Sources}

In order for us to analyze the depth resolution of an OSH system, two point sources located at $\left(x_{1}, y_{1}, z_{1}\right)$ and $\left(x_{2}, y_{2}, z_{2}\right)$, where $z_{1} \neq z_{2}$, are used. This object can be represented mathematically as $f(x, y ; z)=$ $\delta\left(x-x_{1}, y-y_{1}\right) \delta\left(z-z_{1}\right)+\delta\left(x-x_{2}, y-y_{2}\right) \delta\left(z-z_{2}\right)$. The hologram, as computed from Eq. (יㅗ), is

$$
g(x, y)=h\left(x-x_{1}, y-y_{1} ; z_{1}\right)+h\left(x-x_{2}, y-y_{2} ; z_{2}\right) .
$$

Note that this measurement is a sum of two shifted PSF functions. Because they are not orthogonal to each other,

$$
\iint_{-\infty}^{\infty} h\left(x-x_{1}, y-y_{1} ; z_{1}\right) h^{*}\left(x-x_{2}, y-y_{2} ; z_{2}\right) \mathrm{d} x \mathrm{~d} y \neq 0,
$$

and therefore generally no reconstruction method can completely eliminate the contribution from one object section to the other. Some residual signals from the $z_{2}$ plane will exist in the reconstruction for the section at $z_{1}$. If we use the conventional method for this object sectional image reconstruction, we obtain

$$
\begin{aligned}
f_{\text {est }}\left(x, y ; z_{1}\right)= & \delta\left(x-x_{1}, y-y_{1}\right) \delta\left(z-z_{1}\right)-j \frac{k}{2 \pi\left(z_{2}-z_{1}\right)} \\
& \times \exp \left\{j \frac{k}{2\left(z_{2}-z_{1}\right)}\left[\left(x-x_{2}\right)^{2}+\left(y-y_{2}\right)^{2}\right]\right\} \\
= & \delta\left(x-x_{1}, y-y_{1}\right) \delta\left(z-z_{1}\right) \\
& +h\left(x-x_{2}, y-y_{2} ; z_{2}-z_{1}\right),
\end{aligned}
$$

where the term $h\left(x-x_{2}, y-y_{2} ; z_{2}-z_{1}\right)$ is the residue signal in the estimation.

Note that the amplitude of the residue signal, $k /\left[2 \pi\left(z_{2}-z_{1}\right)\right]=1 /\left[\lambda\left(z_{2}-z_{1}\right)\right]$, determines the $\mathrm{OSH}$ system depth resolution. As long as this is significantly smaller than the original object signal, the two sections in $z_{1}$ and $z_{2}$ can be distinguished from each other. In such a case, we consider that an OSH system has a depth resolution equal to or smaller than $\left|z_{2}-z_{1}\right|$.

In the following study, we use the residue signal amplitude to analyze the OSH system depth resolution. We first find the connection between the depth resolution and the source wavelength $\lambda$. We consider the following case: the first point source location is fixed at $z_{1}=0$ plane, the reconstructed signal is for the first object section, and the source wavelength can be changed among $\lambda=0.5,1,2$.

With these parameters, we plot the residual signal amplitude $1 /\left[\lambda\left(z_{2}-z_{1}\right)\right]$ as a function of $z_{2}$ (which is bigger than $z_{1}$ ) in Fig. $\underline{2}$. As $z_{2}$ increases, the residue signal has a smaller amplitude, because the two sections in $z_{1}$ and $z_{2}$ do not interfere with each other much, as expected. We can also observe that, when $z_{2}$ increases by a fixed value $\Delta z_{2}, 1 /\left[\lambda\left(z_{2}-z_{1}\right)\right]$ decreases faster for $\lambda=0.5$ than for $\lambda=2$. This means that for an OSH system using a source with a shorter wavelength $\lambda$, the residual signal decreases faster when the section in $z_{2}$ plane moves away from $z_{1}$. Hence, such an OSH system has a smaller depth resolution compared with a system using a longer wavelength source. As for the connection between

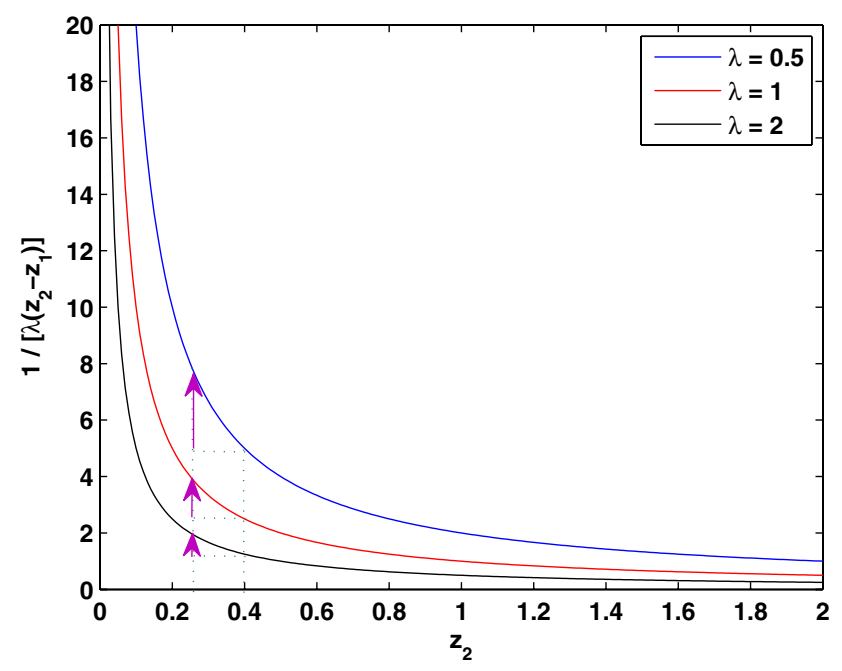

Fig. 2. (Color online) Function $1 /\left[\lambda\left(z_{2}-z_{1}\right)\right]$ versus $z_{2}$ for $\lambda=$ $0.5,1,2$ with $z_{1}=0$. 
the depth resolution and the first section position $z_{1}$, we notice that when $\left(z_{2}-z_{1}\right)$ is fixed, the residue signal amplitude $1 /\left[\lambda\left(z_{2}-z_{1}\right)\right]$ does not change as $z_{1}$ varies. However, this is based on an assumption that the hologram recorded for measurement is obtained using infinite size source beams. In the following, we demonstrate that the residual signal amplitude indeed changes as $z_{1}$ varies.

We use the conventional method to reconstruct object section in the $z$ plane for two points at $(x, y, z)=$ $\left(0,0, z_{1}\right)$ and $(x, y, z)=\left(0,0, z_{2}\right)$ positions. The hologram in the range of $-500 \mu \mathrm{m} \leq x, y \leq 500 \mu \mathrm{m}$ is recorded. The estimated signal at $(x, y)=(0,0)$ is plotted as a function of $z$ in Fig. $\underline{3}$, where $\left(z_{2}-z_{1}\right)$ is fixed at $10 \mathrm{~mm},\left(z_{1}, \lambda\right)$ is $(65 \mathrm{~mm}, 550 \mathrm{~nm}),(65 \mathrm{~mm}$, $480 \mathrm{~nm})$, and (35 mm,550 nm) in Figs. 3(a), (b), and (c), respectively. It can be observed that the two points barely resolved in Fig. 3(a) become two distinguishable peaks in (b) and (c) as either $z_{1}$ or $\lambda$ decreases. This is because the OSH system PSF $h(x, y ; z)$ is a complex FZP, and therefore in (b) and (c) the transition between opaque and transparent zones are faster, which means the FZP has a shorter focal length and depth of focus. Put another way, as shown in Fig. 4, the OSH PSF or the complex FZP in the observation area has more opaque and transparent zones when $z$ decreases. In an OSH system, it means the system has an improved depth resolution because the object in an axial plane different from the FZP focal plane is easier to be out of focus. Therefore, employing a shorter wavelength source or moving the object toward the system improves the OSH system depth resolution. However, the former idea is restricted by the availability of a suitable light source, while the latter is constrained by the minimum distance between the object and the system [3]. To overcome these restrictions, new strategies are required for resolution enhancement. Note that an OSH system can be considered as a set of FZPs while each FZP images object in one $z$ plane for measurement. By changing the system source wavelength, we can have distinctive sets of FZPs for 3D object imaging. Using these multiple sets of imaging measurements, we expect to have a better system reconstruction performance. This motivates us to use a dual-wavelength source for system depth resolution enhancement. (a)

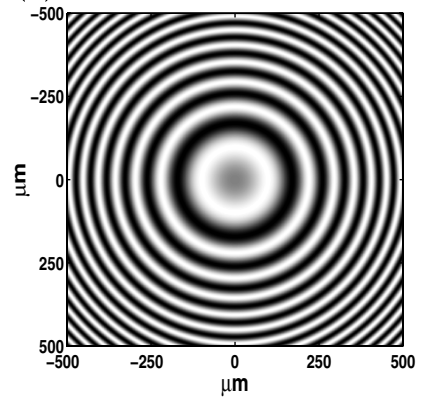

(b)

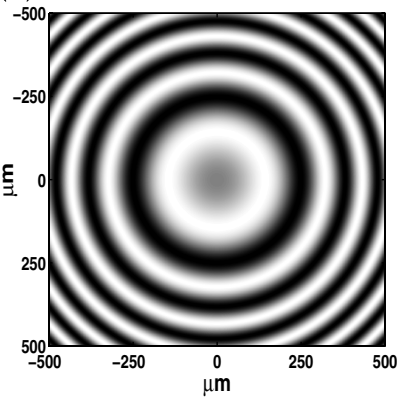

Fig. 4. Real part of an OSH system PSF using Eq. (1) when (a) $z=35 \mathrm{~mm}$, (b) $z=75 \mathrm{~mm}$. The source wavelength is $\lambda=550 \mathrm{~nm}$.

\section{OSH System with a Dual-Wavelength Source}

In this section we describe a DW-OSH system, which we then model with a linear system. This is followed by a description of how to solve the sectioning problem in DW-OSH, where the Fourier domain CG method is used.

\section{A. OSH System with a Source Working at Two Wavelengths}

A DW-OSH system is equipped with a source that works at two wavelengths $\lambda_{1}$ and $\lambda_{2}$ sequentially. Options for such a laser source include a wavelengthswitchable laser and a multiline tuning laser such as a Krypton or Argon Ion laser emitting at several wavelengths in the visible and ultraviolet spectrum. A wavelength-switchable laser is used in this work.

The system diagram for a DW-OSH system is almost the same as Fig. 1, except for the source. Note that the total data acquisition time for the DW-OSH will be doubled compared with SW-OSH, which potentially limits its applicability to in vivo imaging or applications in which the samples can be easily overexposed by the source. To cope with this issue, we need to increase the data acquisition rate in DW-OSH, as much as doubling to maintain the same overall data capture time. This requires that the AOFS, scanning mirror, and data acquisition instrument should all be high-speed. A consequence, however, is that in each DW-OSH measurement the object signal is reduced by half because of the
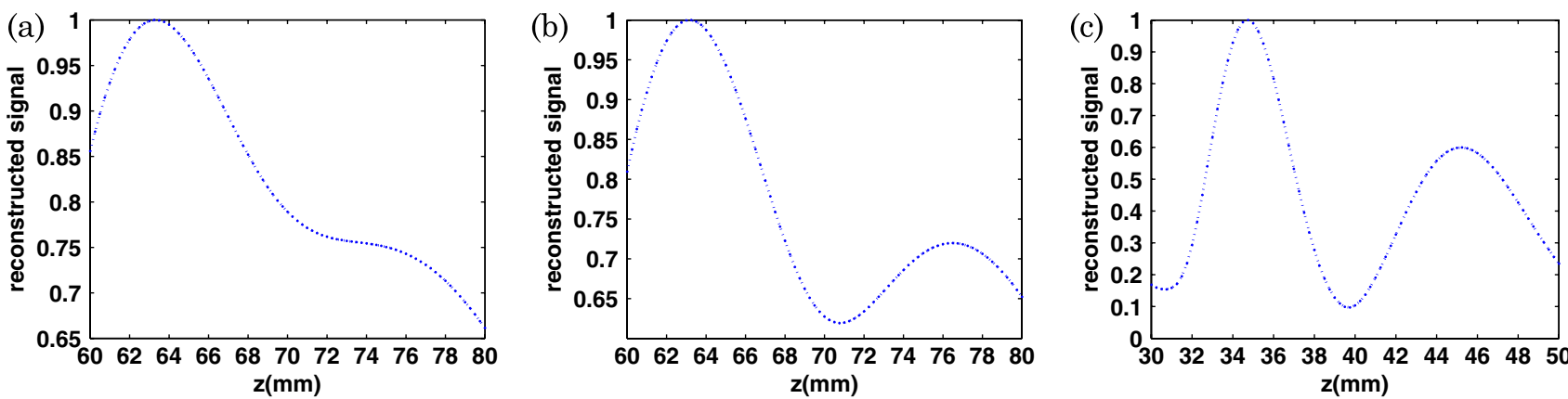

Fig. 3. (Color online) Reconstruction signal at $(x, y)=(0,0)$ versus $z$. For (a), $z_{1}=65 \mathrm{~mm}, z_{2}=75 \mathrm{~mm}$, and $\lambda=550 \mathrm{~nm}$; for (b), $z_{1}=65 \mathrm{~mm}, z_{2}=75 \mathrm{~mm}$, and $\lambda=480 \mathrm{~nm}$; while for (c), $z_{1}=35 \mathrm{~mm}, z_{2}=45 \mathrm{~mm}$, and $\lambda=550 \mathrm{~nm}$. 
reduced exposure. The detector needs to work with a larger bandwidth, leading to more detector noise $[10,11]$. Specifically, if we assume the detector exposure time in SW-OSH for one measurement is $T_{0}$, then the noise energy is proportional to $\sigma_{0}^{2} / T_{0}$, where $\sigma_{0}^{2}$ is the noise energy per bandwidth. Because the detector exposure time in DW-OSH is halved, the noise variance in each measurement increases to $2 \sigma_{0}^{2} / T_{0}$ [12]. Thus, the decreased object signal and doubled measurement noise together degrade the signal-tonoise ratio (SNR) in DW-OSH.

To model the DW-OSH system measurement process, with the purpose of facilitating sectional image reconstruction, which we will describe in the next section, we first discretize the number of sections to $M$. The SW-OSH imaging equation in Eq. (4) can be written as [6]

$$
g(x, y)=\sum_{i=1}^{M}\left(f\left(x, y ; z_{i}\right) * h\left(x, y ; z_{i}\right)\right),
$$

while the equivalent equation in the Fourier domain is

$$
\mathcal{F}\{g(x, y)\}=\sum_{i=1}^{M}\left(\mathcal{F}\left\{f\left(x, y ; z_{i}\right)\right\} \mathcal{F}\left\{h\left(x, y ; z_{i}\right)\right\}\right) .
$$

In a DW-OSH system, $g(x, y)$ is replaced by $g_{1}(x, y)$ and $g_{2}(x, y)$, corresponding to $h_{1}\left(x, y ; z_{i}\right)$ and $h_{2}\left(x, y ; z_{i}\right)$, respectively, for the two source wavelengths.

Our next step is to convert the above to a matrix formulation. We represent the lexicographically ordered $2 \mathrm{D}$ system measurement $\mathcal{F}\left\{g_{1}(x, y)\right\}$ as a vector $\mathbf{g}_{1}$ of size $N \times 1$. Similarly, $\mathcal{F}\left\{f\left(x, y ; z_{i}\right)\right\}$ is represented as a vector $\mathbf{f}\left(z_{i}\right)$ of size $N \times 1$. The product $\mathcal{F}\left\{f\left(x, y ; z_{i}\right)\right\} \mathcal{F}\left\{h_{1}\left(x, y ; z_{i}\right)\right\}$ becomes $\mathrm{H}_{1}\left(z_{i}\right) \mathbf{f}\left(z_{i}\right)$, where the matrix $\mathrm{H}_{1}\left(z_{i}\right)$ is diagonal with elements representing $\mathcal{F}\left\{h_{1}\left(x, y ; z_{i}\right)\right\}$. The system also contains noise, which we assume to be multidimensional additive white Gaussian represented by $\mathbf{n}_{1}$, again of size $N \times 1$. Accordingly, the imaging equation for this first wavelength is given by

$$
\begin{aligned}
\mathbf{g}_{1}=\mathrm{H}_{1} \mathbf{f}+\mathbf{n}_{1}= & {\left[\mathrm{H}_{1}\left(z_{1}\right) \mathrm{H}_{1}\left(z_{2}\right) \cdots \mathrm{H}_{1}\left(z_{M}\right)\right] } \\
& \times\left[\begin{array}{c}
\mathbf{f}\left(z_{1}\right) \\
\mathbf{f}\left(z_{2}\right) \\
\vdots \\
\mathbf{f}\left(z_{M}\right)
\end{array}\right]+\mathbf{n}_{1} .
\end{aligned}
$$

Note that we use the Fourier domain because of the sparsity of the matrix $\mathrm{H}_{1}\left(z_{i}\right)$, which will benefit the speed of convergence and the memory requirement in the sectioning process. This makes solving a large-dimensional sectioning problem feasible.

When we include the contribution of the second wavelength, the imaging equation becomes

$$
\begin{aligned}
\mathbf{g} & =\left[\begin{array}{l}
\mathbf{g}_{1} \\
\mathbf{g}_{2}
\end{array}\right] \\
& =\left[\begin{array}{llll}
\mathrm{H}_{1}\left(z_{1}\right) & \mathrm{H}_{1}\left(z_{2}\right) & \cdots & \mathrm{H}_{1}\left(z_{M}\right) \\
\mathrm{H}_{2}\left(z_{1}\right) & \mathrm{H}_{2}\left(z_{2}\right) & \cdots & \mathrm{H}_{2}\left(z_{M}\right)
\end{array}\right]\left[\begin{array}{c}
\mathbf{f}\left(z_{1}\right) \\
\mathbf{f}\left(z_{2}\right) \\
\vdots \\
\mathbf{f}\left(z_{M}\right)
\end{array}\right]+\left[\begin{array}{l}
\mathbf{n}_{1} \\
\mathbf{n}_{2}
\end{array}\right] \\
& =\mathrm{H} \mathbf{f}+\mathbf{n},
\end{aligned}
$$

where $\mathbf{g}$ of size $2 N \times 1$ is the measurement vector, $\mathrm{H}$ of size $2 N \times M N$ is the system response matrix, $\mathbf{f}$ of size $M N \times 1$ is the object vector, and $\mathbf{n}$ of size $2 N \times 1$ is the noise vector. Note that the physical size of an object does not change with the OSH system source wavelength. The vector $\mathbf{f}$ represents the original object using a fixed sampling step size regardless of the imaging system parameters. Therefore, with a sufficiently fine sampling step, we expect to observe a difference in lateral resolution represented by a different number of reconstruction pixels using different various wavelengths. However, this effect is not our focus and does not conflict with our measurement model and hence is not discussed further in the following sections.

\section{B. Conjugate Gradient Method for Inverse Imaging}

To reconstruct object sections, we need to recover the object vector $\mathbf{f}$ given the measurement in $\mathbf{g}$, which is an inverse problem. A broadly used method is to find the solution to the following minimization problem,

$$
\mathbf{f}_{\mathrm{est}}=\arg \min _{\mathbf{f}}\|\mathbf{H} \mathbf{f}-\mathbf{g}\|_{2}^{2}+\rho\|\mathbf{C f}\|_{2}^{2},
$$

where $\mathbf{f}_{\text {est }}$ is the estimated object vector, $\rho>0$ is a penalty parameter, and $\mathbf{C}$ is a diagonal regularization matrix, such as a Laplacian operator that is used in this work [13], while other forms are also possible [14]. Therefore, the cost function of this minimization problem consists of two terms, one for the measurement domain error and the other for the regularization of the minimizer. The solution to this problem can be obtained analytically as

$$
\left(\mathrm{H}^{+} \mathrm{H}+\rho \mathrm{C}^{+} \mathrm{C}\right) \mathbf{f}_{\mathrm{est}}=\mathrm{H}^{+} \mathbf{g},
$$

where $\mathrm{H}^{+}$indicates the conjugate transpose of $\mathrm{H}$.

To solve this linear system of equations, we use the CG method because the search directions in this iterative technique are a set of orthogonal directions, and hence provide a faster convergence than the steepest descent [15]. In fact, the convergence depends on the condition number of $\left(\mathrm{H}^{+} \mathrm{H}+\rho \mathrm{C}^{+} \mathrm{C}\right)$. If this matrix is sparse, which is the case as we use the Fourier domain formulation, the computational cost will be less. A special case is that for a two-section object, $\left(\mathrm{H}^{+} \mathrm{H}+\rho \mathrm{C}^{+} \mathrm{C}\right)$ is not only symmetric, but also is a block $2 \times 2$ symmetric matrix equal to 
$\left[\begin{array}{cc}\sum_{i=1}^{2} \mathrm{H}_{i}^{+}\left(z_{1}\right) \mathrm{H}_{i}\left(z_{1}\right) & \sum_{i=1}^{2} \mathrm{H}_{i}^{+}\left(z_{1}\right) \mathrm{H}_{i}\left(z_{2}\right) \\ \sum_{i=1}^{2} \mathrm{H}_{i}^{+}\left(z_{2}\right) \mathrm{H}_{i}\left(z_{1}\right) & \sum_{i=1}^{2} \mathrm{H}_{i}^{+}\left(z_{2}\right) \mathrm{H}_{i}\left(z_{2}\right)\end{array}\right]+\rho \mathrm{C}^{+} \mathrm{C}$.

Special CG methods have been studied for such a symmetric matrix [16]. Thus, in the case of a twosection object, we choose the CG method referred to as block Jacobi-type restrictive preconditioned CG (BJ-RPCG), the details of which are given in [16].

\section{Simulations}

For our simulations we assume the source in SW$\mathrm{OSH}$ functions at wavelength $\lambda_{1}=543 \mathrm{~nm}$, while in DW-OSH the source functions at two wavelengths, with $\lambda_{1}=543 \mathrm{~nm}$ and $\lambda_{2}=633 \mathrm{~nm}$. These wavelength values $\lambda_{1}$ and $\lambda_{2}$ are two of the five working wavelengths in a commercially available wavelength-switchable laser. The source wavelength of SW-OSH is chosen as $543 \mathrm{~nm}$ instead of $633 \mathrm{~nm}$ because the reconstruction result will be degraded as we discussed in Section 2.B. An object of two sections located at $z_{1}=60 \mathrm{~mm}$ and $z_{2}=60.0025 \mathrm{~mm}$ is assumed in the study. The distance between the two sections is therefore $\Delta z=2.5 \mu \mathrm{m}$. Each object section has size $401 \times 401$ with an 8 bit dynamic range.

Figures 5(a) and (b) present the two sections used for our study. Note that the object vector $f$ has a length of $2 \cdot 401^{2}=321,602$, and the matrix $\left(\mathrm{H}^{+} \mathrm{H}+\rho \mathrm{C}^{+} \mathrm{C}\right)$ therefore has size $321,602 \times 321,602$. However, the matrix is never actually stored in practice, and the matrix-vector computation is not directly implemented. The matrix $\left(\mathrm{H}^{+} \mathrm{H}+\rho \mathrm{C}^{+} \mathrm{C}\right)$ can be diagonalized by the Fourier basis, and therefore only the diagonal elements, i.e., the Fourier coefficients, are needed in the calculations. The cosine and sine holograms at $\lambda_{1}$ are presented in Figs. 5(c) and (d). The measurement SNR for SW-OSH, defined as an average of the object pixel energy divided by the detector noise variance, $\|\mathbf{f}\|_{2}^{2} /(M N) / \sigma^{2}$, is computed to be $40.35 \mathrm{~dB}$. Because of the shortened exposure time, the SNR of DW$\mathrm{OSH}$ is only $31.32 \mathrm{~dB}$, which is about $9 \mathrm{~dB}$ lower.

To reconstruct the two object sections, we compare three methods: (1) the conventional method with SWOSH measurements, (2) BJ-RPCG for SW-OSH, and (3) BJ-RPCG for DW-OSH. The section depth information $z_{i}$ is assumed to be known and otherwise can be obtained with techniques such as in [17]. In Fig. 6, the first row presents the reconstructions at $z_{1}$ using these three methods, respectively, while the second row shows the corresponding results at $z_{2}$. It can be observed that with SW-OSH, neither the conventional method nor the inverse imaging method using BJ-RPCG provides acceptable sectioning results. Both contain significant residual signals from the other section. As a result, we consider that the depth resolution in SW-OSH is larger than the distance between the two sections, i.e., $\Delta z=2.5 \mu \mathrm{m}$. On the other hand, with DW-OSH system measurements the reconstruction using BJ-RPCG significantly suppresses the residual signals. Thus, we consider that the depth resolution in DW-OSH is equal to or smaller than $2.5 \mu \mathrm{m}$.

For the next study, we first increase the detector noise and repeat the above experiment for DW-OSH, where the measurement SNR reduces by as much as $10 \mathrm{~dB}$. Figures 7(a) and (d) show the sectioning results for $z_{1}$ and $z_{2}$, respectively. Comparing them with the results in Figs. 6(c) and (f), we observe that as noise increases, the section estimations present larger background noise and residual signals, as expected. To improve these sectioning results, we then enlarge the wavelength difference between $\lambda_{1}$ and $\lambda_{2}$ by using $\lambda_{1}=400 \mathrm{~nm}$ and $\lambda_{2}=700 \mathrm{~nm}$. Figures $7(\mathrm{~b})$ and (e) show the corresponding results. The background noise and residual signals are reduced accordingly. A second method to improve sectioning result is to reduce the $z_{1}$ value, as discussed in Section 2.B.. The section reconstructions when $z_{1}$ is reduced from $60 \mathrm{~mm}$ to $0.1 \mathrm{~mm}$ are presented in Figs. 7(c) and (f). They have better contrast than the reconstructions in (a) and (d) but are not as good as (b) and (e). To understand this, we present in Fig. 8 the real part of three OSH system PSFs for $z_{1}$. The upper row is for source wavelength $\lambda_{1}$, and the lower row for $\lambda_{2}$. For a short axial distance $\Delta z=2.5 \mu \mathrm{m}$, PSF for $z_{2}$ is almost the same as that for $z_{1}$. As discussed in Section 2 , the fringe pattern in PSF becomes denser as $z$ decreases. However, the difference between the two PSFs using two source wavelengths is not increased a lot. Therefore, reducing $z$ does not provide much help for the second set of FZPs to collect more object information. On the other hand, if we increase the difference in the two source wavelengths such as in Fig. 8(b), the difference between the two PSFs is more pronounced. Hence a better reconstruction is expected, which is consistent with the results in Fig. 7. A conclusion we can draw here is that enlarging the (a)

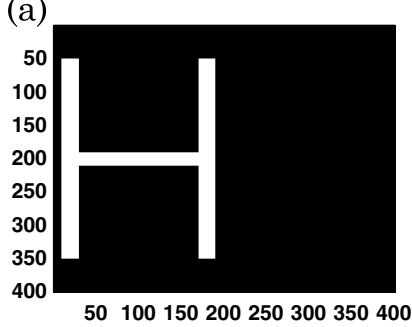

(b)

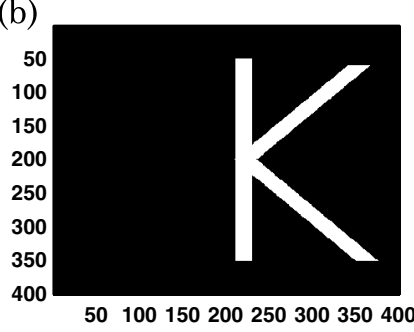

(c)

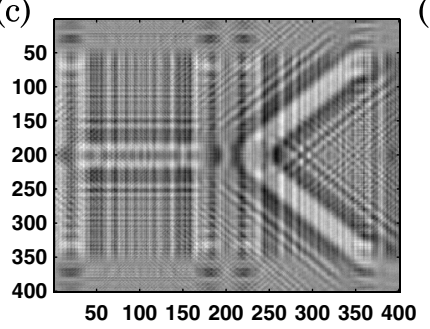

(d)

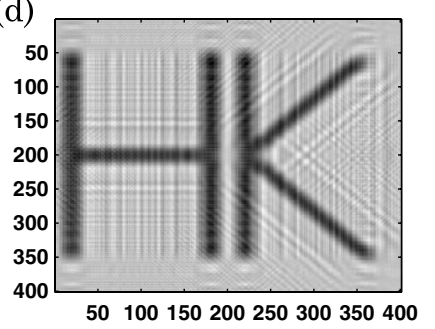

Fig. 5. Original object sections located at (a) $z_{1}$ and (b) $z_{2}$ planes. (c) Cosine and (d) sine holograms recorded using a source working at wavelength $\lambda_{1}=543 \mathrm{~nm}$ when the measurement SNR is $40.35 \mathrm{~dB}$. 
(a)

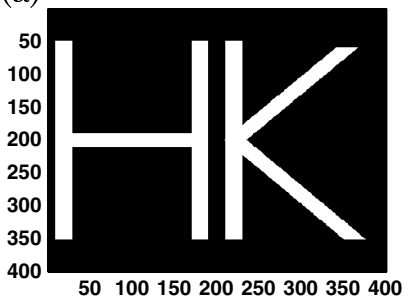

(d)

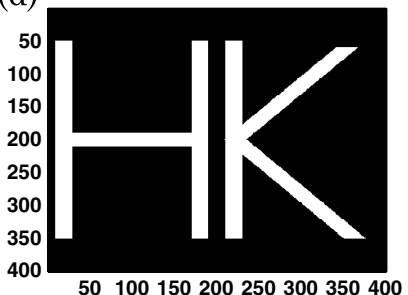

(b)
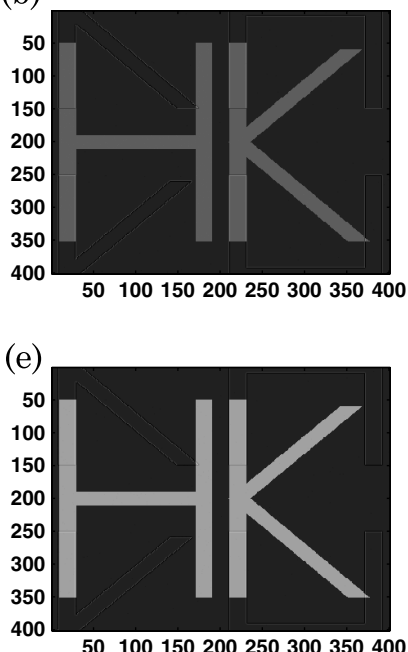

(c)

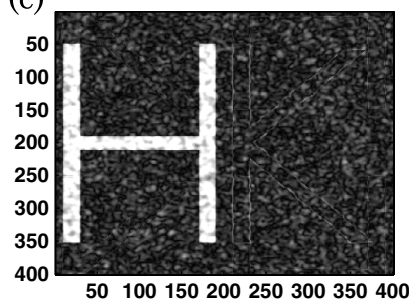

(f)

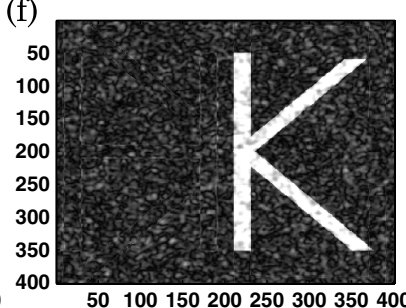

Fig. 6. Sectioning results using the conventional method with SW-OSH measurements for (a) $z_{1}$ and (d) $z_{2}$ planes, using BJ-RPCG method with SW-OSH measurements for (b) $z_{1}$ and (e) $z_{2}$ planes, and using BJ-RPCG method with DW-OSH measurements for (c) $z_{1}$ and (f) $z_{2}$ planes. The source wavelength for SW-OSH is $\lambda_{1}=543 \mathrm{~nm}$. The source wavelengths for DW-OSH are $\lambda_{1}=543 \mathrm{~nm}$ and $\lambda_{2}=633 \mathrm{~nm}$. The measurement SNR is $40.35 \mathrm{~dB}$ for SW-OSH and $31.32 \mathrm{~dB}$ for DW-OSH.

difference between the two source wavelengths is a more effective method for OSH system depth resolution enhancement.

\section{OSH System Simulator}

To facilitate further investigation into the depth resolution enhancement for OSH, we present in this section a simulator that we have developed. Three kinds of system parameters are considered: (1) the finite diameters of apertures, (2) the finite scanning step size in the $2 \mathrm{D}$ scan process, and (3) thermal, Poisson, and quantization error measurement noise.

The OSH system PSF will change as the diameter of apertures varies. To rederive a more precise system PSF, consider Fig. 9 , which shows an OSH source beam propagating through the system elements, and an object is then detected by a large area detector.
The aperture A1 represents the pinhole or open aperture that is used to generate a plane or spherical wave for object illumination. It is located at the front focal plane of lens L1. A second aperture, A2, is located at $z$ away from the back focal plane of L1, which is the plane just before the object. It is used to ensure that both spherical and plane source waves have the same diameters for illumination. We further assume that the two apertures the lens aperture of L 1 all are circular. In addition, an object is assumed to be just behind $\mathrm{A} 2$, at a distance $z$ away from the back focal plane of L1. Instead of using a scanning mirror, the 2D scan process is achieved by assuming that the object is on a moving stage controlled by a motion controller [3]

We can now derive the wavefront $U_{z}(x, y)$ after $\mathrm{A} 2$ but just before object. This allows us to then find the OTF and the PSF using Fresnel approximation [18]. (a)

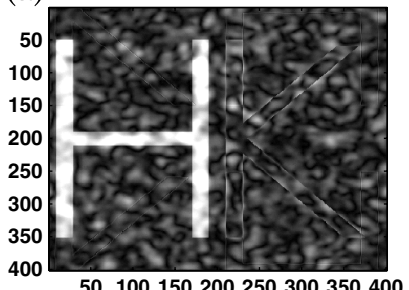

(d)

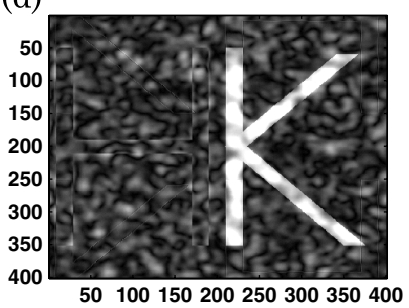

(b)

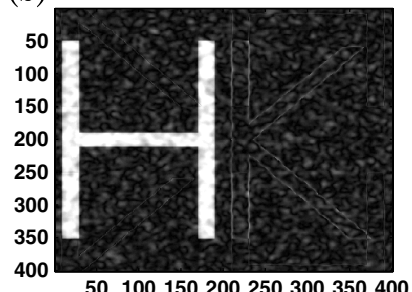

(e)

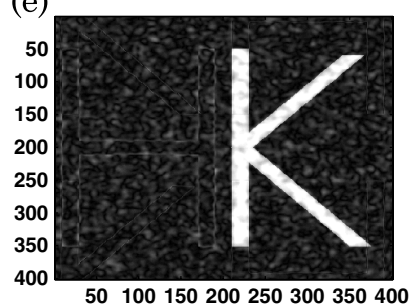

(c)

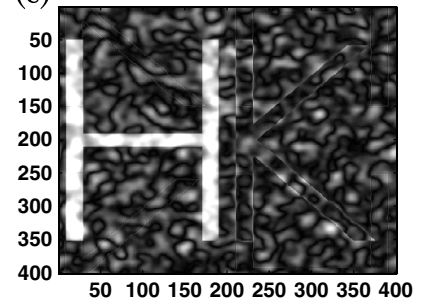

(f)

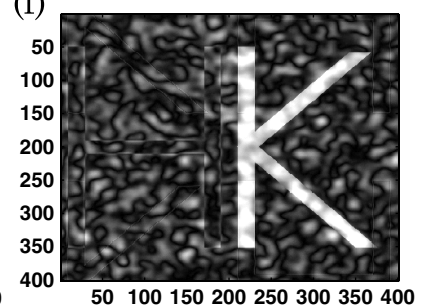

Fig. 7. Sectioning results for $z_{1}$ and $z_{2}$ planes using BJ-RPCG with DW-OSH measurements. The first row shows the results for $z_{1}$ while the second row for $z_{2}$. For (a) and (d), $\lambda_{1}=543 \mathrm{~nm}, \lambda_{2}=633 \mathrm{~nm}$, and $z_{1}=60 \mathrm{~mm}$; for (b) and (e), $\lambda_{1}=400 \mathrm{~nm}, \lambda_{2}=700 \mathrm{~nm}$, and $z_{1}=60 \mathrm{~mm}$; while for (c) and (f), $\lambda_{1}=543 \mathrm{~nm}, \lambda_{2}=633 \mathrm{~nm}$, and $z_{1}=0.1 \mathrm{~mm}$. The measurement SNR is $21.32 \mathrm{~dB}$. 
(a)

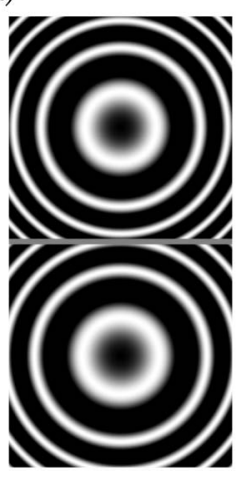

(b)

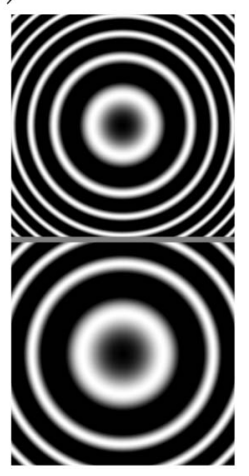

(c)

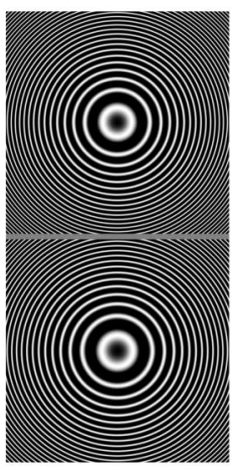

where $\rho=\sqrt{x^{2}+y^{2}} / \lambda f, r$ is the radius of aperture $\mathrm{A} 1$, and $J_{1}$ is the Bessel function of the first kind, order 1 [18]. Using $U_{f}(x, y)$, we can obtain $U_{z}(x, y)$ based on Fresnel approximation [18],

$$
U_{z}(x, y)=\frac{\exp \{j k z\}}{j \lambda z} U_{f}(x, y) * \exp \left\{\frac{j k}{2 z}\left(x^{2}+y^{2}\right)\right\} .
$$

Fig. 8. The real part of the OSH PSF for $z_{1}$ plane, where (a) $\lambda_{1}=543 \mathrm{~nm}, \quad \lambda_{2}=633 \mathrm{~nm}, \quad$ and $z_{1}=60 \mathrm{~mm}$, (b) $\lambda_{1}=400 \mathrm{~nm}, \lambda_{2}=700 \mathrm{~nm}$, and $z_{1}=60 \mathrm{~mm}$; (c) $\lambda_{1}=543 \mathrm{~nm}$, $\lambda_{2}=633 \mathrm{~nm}$, and $z_{1}=10 \mathrm{~mm}$.

$$
\begin{aligned}
\mathcal{F}\left\{U_{z}(x, y)\right\} & =\frac{\exp \{j k z\}}{j \lambda z} \mathcal{F}\left\{U_{f}(x, y)\right\} \mathcal{F}\left\{\exp \left\{\frac{j k}{2 z}\left(x^{2}+y^{2}\right)\right\}\right\} \\
& =\frac{\exp \{j k z\}}{j \lambda z}\left[(\lambda f) t_{A 1}(\lambda f x, \lambda f y)\right]\left(\frac{2 \pi z}{-j k}\right) \exp \left\{\frac{-j z}{2 k}\left[(2 \pi x)^{2}+(2 \pi y)^{2}\right]\right\} \\
& =\frac{2 \pi f \exp \{j k z\}}{k} t_{A 1}^{\prime}(x, y) \exp \left\{\frac{-j z}{2 k}\left[(2 \pi x)^{2}+(2 \pi y)^{2}\right]\right\}
\end{aligned}
$$

To derive $U_{z}(x, y)$, the wavefront $U_{f}(x, y)$ at the back focal plane of $\mathrm{L} 1$ is calculated first. If we use $t_{A 1}(x, y)$ to represent aperture $\mathrm{A} 1$, then $U_{f}(x, y)$ can be written as $[18]$

$$
\begin{aligned}
U_{f}(x, y)= & \frac{1}{j \lambda f} \iint_{-\infty}^{\infty} t_{A 1}(u, v) P(u+x, v \\
& +y) \exp \left\{-j \frac{2 \pi}{\lambda f}(u x+v y)\right\} \mathrm{d} u \mathrm{~d} v
\end{aligned}
$$

where $P(x, y)$ is the aperture function of L1 with diameter $D_{1}$. For a pinhole aperture, its diameter is much smaller than this. Therefore, $P(x, y)$ can be assumed unity in the integration. For an open aperture, we cannot directly make this assumption. However, if the diameter of aperture A2 is much smaller than $D_{1}$ or if we only use the wavefront at a small paraxial angle for object illumination, we can still assume $P(x, y)=1$. With this assumption, and dropping the constant in front of the integration, we simplify the wavefront $U_{f}(x, y)$ as

$$
\begin{aligned}
U_{f}(x, y) & =\iint_{-\infty}^{\infty} t_{A 1}(u, v) \exp \left\{-j 2 \pi\left(u \frac{x}{\lambda f}+v \frac{y}{\lambda f}\right)\right\} \mathrm{d} u \mathrm{~d} v \\
& =r \frac{J_{1}(2 \pi r \rho)}{\rho}
\end{aligned}
$$

where

$$
t_{A 1}^{\prime}(x, y)=\left\{\begin{array}{cc}
1 & \sqrt{x^{2}+y^{2}}<r / \lambda f \\
0 & \text { otherwise }
\end{array}\right.
$$

Similarly, the Fourier transform of $U_{z}^{*}(x, y)$ is

$$
\begin{aligned}
\mathcal{F}\left\{U_{z}^{*}(x, y)\right\}= & \frac{2 \pi f \exp \{-j k z\}}{k} t_{A 1}^{\prime}(x, y) \\
& \times \exp \left\{\frac{j z}{2 k}\left[(2 \pi x)^{2}+(2 \pi y)^{2}\right]\right\} .
\end{aligned}
$$

The $U_{z}(x, y)$ above is the wavefront obtained from one source beam, while in OSH, two source beams, being plane wave and spherical wave, are used for object illumination. If we use $U_{P}(x, y)$ and $U_{S}(x, y)$ to represent the plane and spherical waves just before the object, respectively, then the OTF of the OSH system is $\left[\mathcal{F}\left\{U_{P}(x, y)\right\} \mathcal{F}\left\{U_{S}^{*}(x, y)\right\}\right]^{*}$ [1]. However, as shown in Fig. 9 , there is another circular aperture A2 with radius $r_{A 2}$. Using $t_{A 2}(x, y)$ to represent this aperture and ignoring the scaling factor in $\mathcal{F}\left\{U_{P}(x, y)\right\}$ and $\mathcal{F}\left\{U_{S}^{*}(x, y)\right\}$, we can compute the complete OTF with 


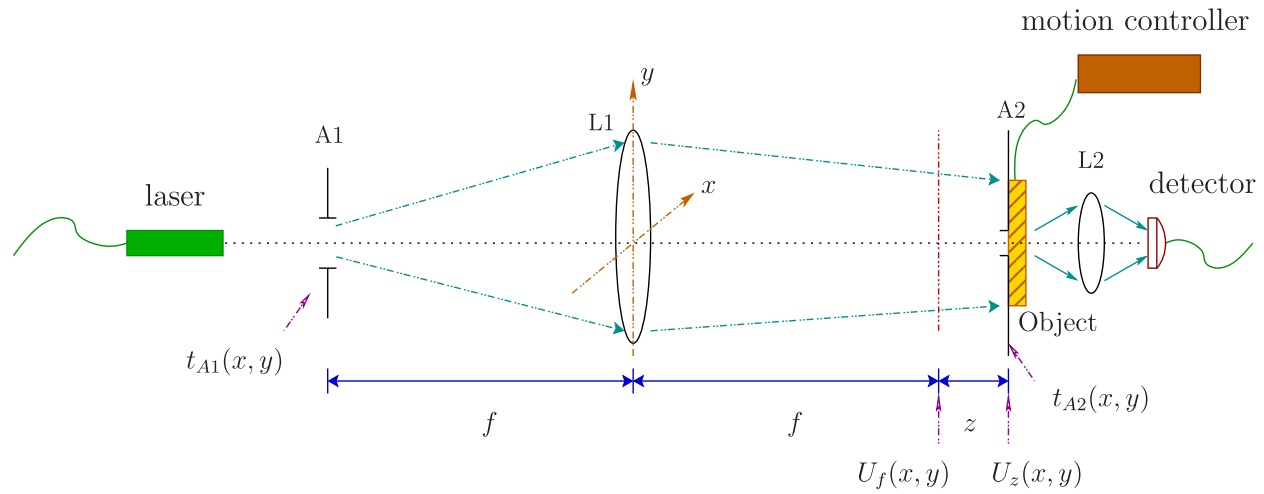

Fig. 9. (Color online) The system diagram for one source beam (plane wave or spherical wave) propagating from the source to the detector.

$$
\begin{aligned}
\mathrm{OTF}(x, y ; z)= & {\left[\mathcal{F}\left\{U_{P}(x, y)\right\} * \mathcal{F}\left\{U_{S}^{*}(x, y)\right\}\right]^{*} } \\
& * \mathcal{F}\left\{t_{A 2}(x, y)\right\} \\
= & t_{P}^{\prime}(x, y) \exp \left\{\frac{j z}{2 k}\left[(2 \pi x)^{2}+(2 \pi y)^{2}\right]\right\} \\
& * t_{S}^{\prime}(x, y) \exp \left\{\frac{-j z}{2 k}\left[(2 \pi x)^{2}+(2 \pi y)^{2}\right]\right\} \\
& * \mathcal{F}\left\{t_{A 2}(x, y)\right\},
\end{aligned}
$$

where $t_{P}^{\prime}(x, y)$ and $t_{S}^{\prime}(x, y)$ have a similar definition as $t_{A 1}^{\prime}(x, y)$, except that $r$ in $t_{P}^{\prime}(x, y)$ and $t_{S}^{\prime}(x, y)$ are $r_{\text {pin }}$ and $r_{\text {open }}$, representing the radii of the pinhole and the open apertures, respectively.

Next we consider the scanning step size in the 2D scan process. As described earlier, the object is on a movable stage controlled by a motion controller. We assume that the scanning step size is $\Delta s=100 \mathrm{~nm}$. In addition, we also need to set other parameters such as $r_{\text {pin }}, r_{\text {open }}$, lens L1 focal length $f$, and the radius $r_{A 2}$ of aperture A2. They are summarized in Table 1. Using these and Eq. (20), we can obtain the OSH system PSF function.
To see how a simulation with the above equations provides more realistic results, in Fig. 10(a) we present the real (shown on the left) and imaginary (shown on the right) parts of the ideal PSF using Eq. (1) with $z=100 \mu \mathrm{m}$ and $\lambda=543 \mathrm{~nm}$, while (b) to $(d)$ present results from our simulator using Eq. (20). In (b), $r_{\text {open }}=10 \mathrm{~mm}$ and $f=50 \mathrm{~mm}$; in (c), $r_{\text {open }}=20 \mathrm{~mm}$ and $f=50 \mathrm{~mm}$; and in (d), $r_{\text {open }}=$ $20 \mathrm{~mm}$ and $f=150 \mathrm{~mm}$. It can be seen that the fringe patterns are affected by the finite size of aperture A1 and the focal length $f$ of lens L1. The PSF using the OSH simulator has less dense fringes compared with the ideal PSF. Therefore, the complex FZP represented by the PSF function has longer focus depth. We expect that the simulated OSH system has a larger depth resolution than that obtained with the ideal system. Also in (b) to (d), we can observe vignetting because the large ratio of $r_{\text {open }}$ to $f$ causes some source light from the boundary area of the open aperture not to pass through lens L1 to reach the ob-

\begin{tabular}{|c|c|c|}
\hline Function/Parameter & Definition & Value \\
\hline$t_{A 1}(x, y)$ & $\begin{array}{l}\text { function for } \mathrm{A} 1 \text {, } \\
\qquad t_{A 1}(x, y)=\left\{\begin{array}{cc}1 & \sqrt{x^{2}+y^{2}}<r \\
0 & \text { otherwise }\end{array}\right.\end{array}$ & \\
\hline $\begin{array}{l}t_{P}(x, y) \\
r_{\text {pin }} \\
t_{S}(x, y) \\
r_{\text {open }} \\
f \\
t_{A 2}(x, y)\end{array}$ & $\begin{array}{l}\left.\text { pinhole aperture for } U_{P}(x, y) \text { (a specific } t_{A 1}(x, y)\right) \\
\text { radius } r \text { in } t_{P}(x, y) \\
\left.\text { open aperture for } U_{S}(x, y) \text { (another specific } t_{A 1}(x, y)\right) \\
\text { radius } r \text { in } t_{S}(x, y) \\
\text { focus length of lens L1 } \\
\text { function for A2 } \\
\qquad t_{A 2}(x, y)=\left\{\begin{array}{cc}1 & \sqrt{x^{2}+y^{2}}<r_{A 2} \\
0 & \text { otherwise }\end{array}\right.\end{array}$ & $\begin{array}{l}2.5 \mu \mathrm{m} \\
10,20 \mathrm{~mm} \\
50,150 \mathrm{~mm}\end{array}$ \\
\hline $\begin{array}{l}r_{A 2} \\
z \\
\Delta s \\
(\sqrt{N} \times \sqrt{N})\end{array}$ & $\begin{array}{l}\text { radius } r \text { in } t_{A 2}(x, y) \\
\text { distance between object and back focal plane of } \mathrm{L} 1 \\
\text { scanning step size } \\
\text { size of the hologram with } N \text { data points }\end{array}$ & $\begin{array}{l}75 \mu \mathrm{m} \\
100 \mu \mathrm{m} \\
100 \mathrm{~nm} \\
(1000 \times 1000)\end{array}$ \\
\hline
\end{tabular}
ject plane. Because of this, in the following we choose the radius of the open aperture $r_{\text {open }}=20 \mathrm{~mm}$ and the focal length of lens L1 at $f=50 \mathrm{~mm}$.

Table 1. Functions and Parameters for the OSH System Simulator 
(a)

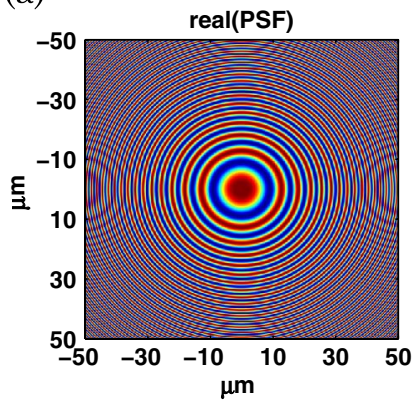

(c)

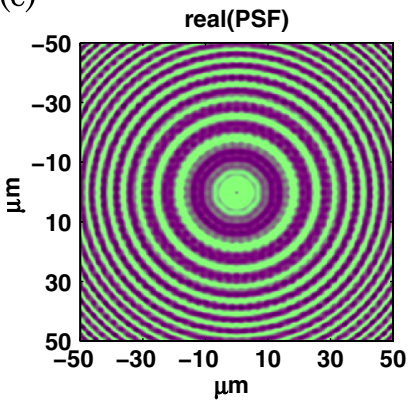

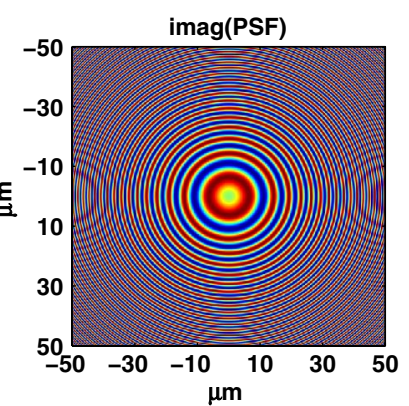

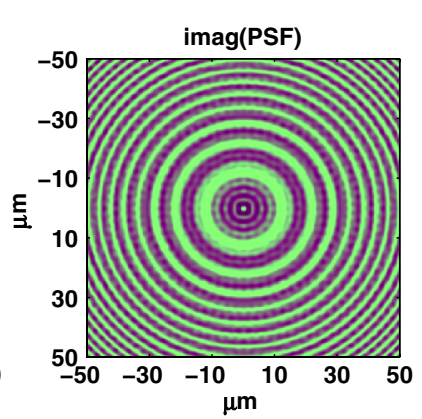

(b)

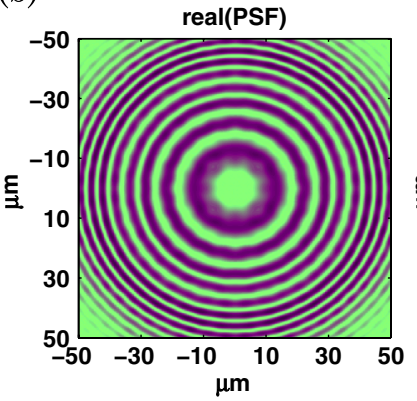

(d)

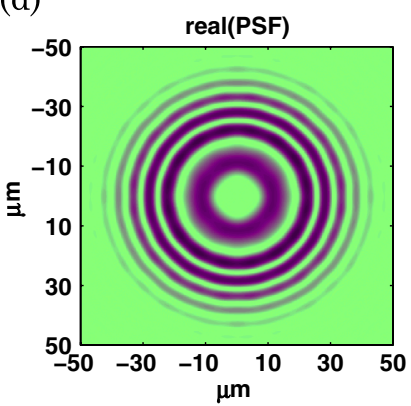

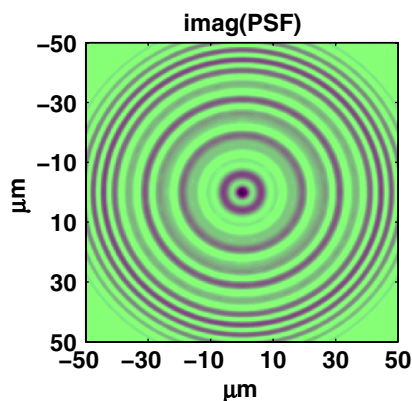

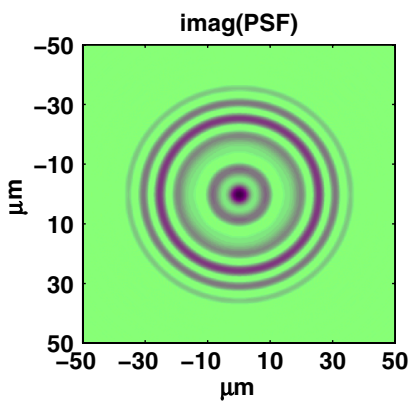

Fig. 10. (Color online) (a) Ideal real and imaginary parts of an OSH system PSF using Eq. (1). The result of our simulator using Eq. (20), (b) when $r_{\text {open }}=10 \mathrm{~mm}$ and $f=50 \mathrm{~mm}$; (c) when $r_{\text {open }}=20 \mathrm{~mm}$ and $f=50 \mathrm{~mm}$; and (d) when $r_{\text {open }}=20 \mathrm{~mm}$ and $f=150 \mathrm{~mm}$. The source wavelength is $\lambda=543 \mathrm{~nm}$. The object section is located at $z=100 \mu \mathrm{m}$.

The third type of system parameters taken into account in the simulator relate to the noise in the imaging system, including thermal, Poisson, and quantization error. Thermal noise originates from the dark current shot noise in the photodetector [19]. We model it as a white Gaussian noise with an energy per bandwidth at $\sigma_{0}^{2}$, where we set $\sigma_{0}=12.75$ when the detector has 8 bit signal dynamic range and the detector exposure time is $T_{0}=1$. As discussed earlier, the thermal noise energy is propor- tional to the working bandwidth of the detector. Therefore the thermal noise variance in a DW-OSH system is twice the noise variance in a SW-OSH system.

Poisson noise comes from the photon counting process in a photodetector. The mean value, denoted by $\bar{p}$, is the detected hologram signal amplitude $g(x, y)$. When it is large, the Poisson noise can be approximated well by a Gaussian distribution $\mathcal{N}\left(\bar{p}, c \bar{p}^{2}\right)$, where $c$ is a constant (we set it at 0.0025 in this (a)

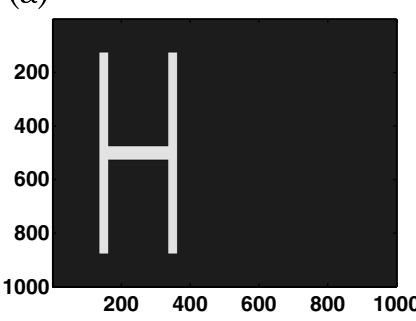

(e)

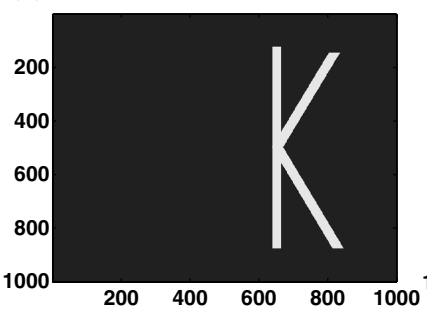

(b)

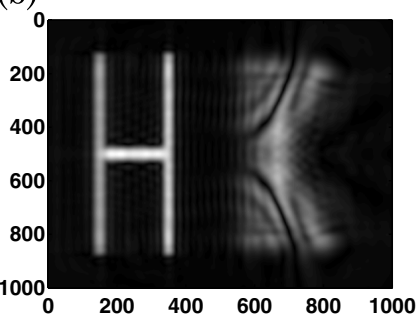

(f)

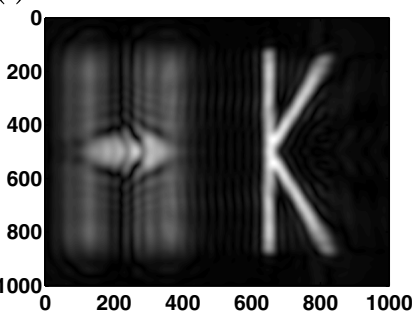

(c)

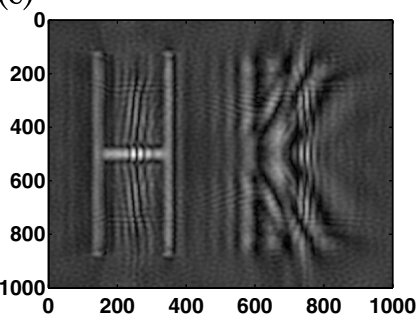

(g)

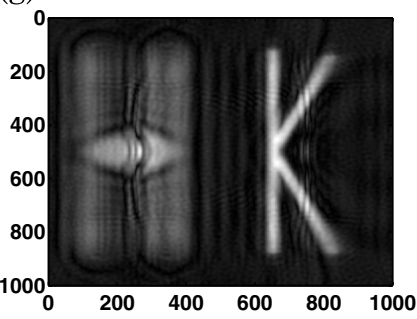

(d)

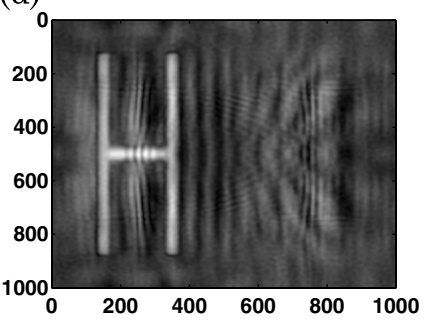

(h)

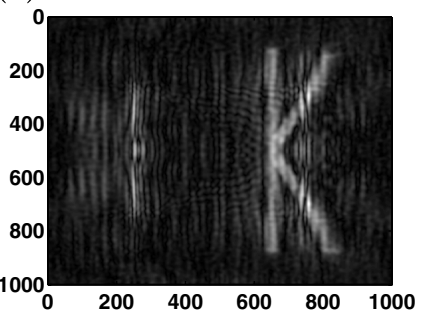

Fig. 11. Original two object sections located at (a) $z_{1}$ and (e) $z_{2}$ planes with $\Delta z=20 \mu \mathrm{m}$. Hologram in area $-50 \mu \mathrm{m} \leq x, y \leq 50 \mu \mathrm{m}$, sampled with $\Delta s=100 \mathrm{~nm}$ is used for reconstruction. Here shows the estimated two sections using the conventional method with SW-OSH measurements for (b) $z_{1}$ and (f) $z_{2}$ planes, using BJ-RPCG method with SW-OSH measurements for (c) $z_{1}$ and (g) $z_{2}$ planes, and using BJ-RPCG method with DW-OSH measurements for $(\mathrm{d}) z_{1}$ and $(\mathrm{h}) z_{2}$ planes. The source wavelength for SW-OSH is $\lambda_{1}=543 \mathrm{~nm}$. The source wavelengths for DW-OSH are $\lambda_{1}=543 \mathrm{~nm}$ and $\lambda_{2}=633 \mathrm{~nm}$. The measurement SNR is $25.34 \mathrm{~dB}$ in SW-OSH and $22.31 \mathrm{~dB}$ in DW-OSH. 
(a)

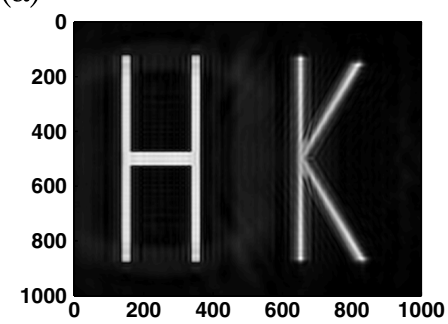

(d)

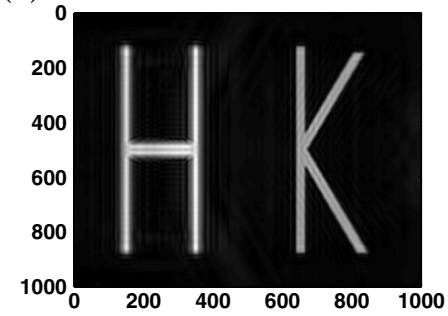

(b)

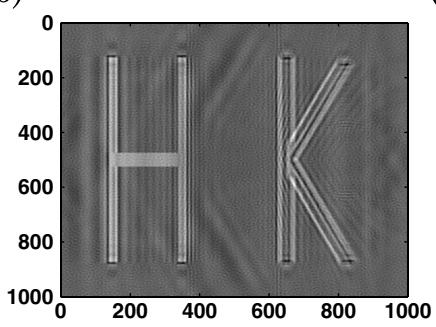

(e)

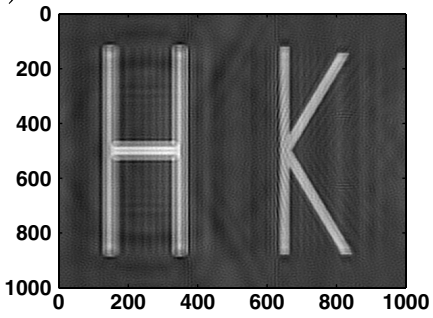

(c)

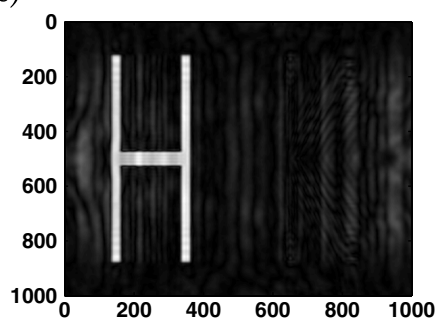

(f)

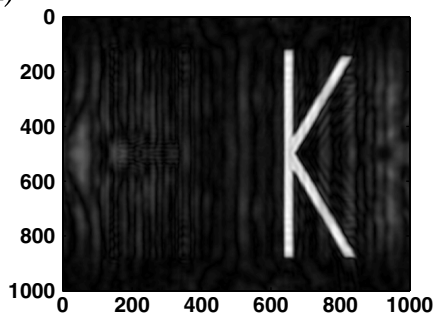

Fig. 12. Original two object sections located at $z_{1}=100 \mu \mathrm{m}$ and $z_{2}=105 \mu \mathrm{m}$ planes. Hologram in area $-100 \mu \mathrm{m} \leq x, y \leq 100 \mu \mathrm{m}$ sampled with $\Delta s=200 \mathrm{~nm}$ is used for reconstruction. Here shows the estimated two sections using the conventional method with SW-OSH measurements for (a) $z_{1}$ and (d) $z_{2}$ planes, using BJ-RPCG method with SW-OSH measurements for (b) $z_{1}$ and (e) $z_{2}$ planes, and using BJ-RPCG method with DW-OSH measurements for (c) $z_{1}$ and (f) $z_{2}$ planes. The source wavelength for SW-OSH is $\lambda_{1}=543$ nm. The source wavelengths for DW-OSH are $\lambda_{1}=543 \mathrm{~nm}$ and $\lambda_{2}=633 \mathrm{~nm}$. The measurement SNR is $25.29 \mathrm{~dB}$ in SW-OSH and $22.36 \mathrm{~dB}$ in DW-OSH.

work). Therefore, we assume that the collected complex hologram signal $\mathrm{Hf}$ as shown in Eq. (11) is a multidimensional Gaussian distributed signal $\mathcal{N}(\mathrm{Hf}, c \Sigma)$, where the diagonal matrix $\Sigma$ has its entries equal to the elementwise square of $\mathrm{Hf}$.

Finally, quantization noise arises from the discrete output levels of a data acquisition process unit in OSH. A 12 bit data acquisition unit is assumed in the simulator. Using this, we have $2^{11}=2048$ levels for either positive or negative measurements. Including these three kinds of noise, the total measurement noise is $\mathbf{n}=\mathbf{n}_{\text {thermal }}+\mathbf{n}_{\text {Poisson }}+\mathbf{n}_{\text {quantization }}$.

Using this simulator, we investigate again the sectioning problem in SW-OSH and DW-OSH systems with source wavelengths $\lambda_{1}=543 \mathrm{~nm}$ and $\lambda_{2}=633 \mathrm{~nm}$. For clarity, the system parameters are repeated here: the pinhole aperture and the open aperture have radius $r_{\text {pin }}=2.5 \mu \mathrm{m}$ and $r_{\text {open }}=$ $20 \mathrm{~mm}$, respectively; aperture A2 has radius $75 \mu \mathrm{m}$; and lens $\mathrm{L} 1$ has focal length $f=50 \mathrm{~mm}$. The scanning step size $\Delta s$ is equal to $100 \mathrm{~nm}$. The thermal noise is a white Gaussian with $\sigma_{0}=12.75$, the Poisson noise is also Gaussian with mean $\mathrm{Hf}$ and covariance matrix $0.0025 \Sigma$, and the quantization error is caused by a 12 bit data acquisition device. The object has two sections, located at $z_{1}=100 \mu \mathrm{m}$ and $z_{2}=120 \mu \mathrm{m}$, with spatial dimension of $1000 \times 1000$ pixels. They are shown in Figs. 11(a) and (e).

Our simulation result shows that the SNR in SWOSH is $25.34 \mathrm{~dB}$, which is about $3 \mathrm{~dB}$ larger than the SNR in DW-OSH at $22.31 \mathrm{~dB}$. This is because we have included the Poisson and the quantization noise in the system measurements, thereby lessening the SNR difference between the two systems. As in Section 4, we use the conventional and the BJ-RPCG methods to reconstruct the two sections. The results from SW-OSH using the conventional method are gi- ven in Fig. 11(b) and (f), while those using BJ-RPCG

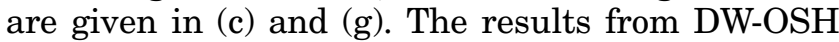
using BJ-RPCG are given in (d) and (h). Comparing the sectioning results in Fig. 11(b) to (d), and (f) to (h), we can observe that the sectioning results using DW$\mathrm{OSH}$ measurements are better than using SW-OSH measurements. To further improve the system performance, we enlarge the area for recording holograms. The number of samples is kept as $1000 \times 1000$, while the sampling step is increased to $200 \mathrm{~nm}$, such that the measurement area is increased from $-50 \mu \mathrm{m} \times 50 \mu \mathrm{m}$ to $-100 \mu \mathrm{m} \times 100 \mu \mathrm{m}$. The distance between the two object sections is decreased to $5 \mu \mathrm{m}$. Using the conventional and the BJ-RPCG methods with SW-OSH and DW-OSH measurements, we have the reconstructed object sections in Fig. 12. Compared with Fig. 11, we can observe that enlarging the hologram recording area helps the DW-OSH system axial resolution performance. Similarly, we expect that reducing the sample step size $\Delta s$ can lead to a similar effect.

\section{Conclusion}

In this work, we have presented a way to increase the $\mathrm{OSH}$ resolution by using two wavelength sources. This is motivated by a careful study of the theoretical resolution using two point sources located at two axial planes, which we then supplant with a more accurate simulator that takes into account further details of the system. We demonstrate that the DW-OSH system depth resolution can reach $2.5 \mu \mathrm{m}$ when the system source wavelengths are at $\lambda_{1}=$ $543 \mathrm{~nm}$ and $\lambda_{2}=633 \mathrm{~nm}$.

This work was supported in part by the Research Grants Council of the Hong Kong Special Administrative Region, China, under Projects HKU 7138/11E, 
and by the University Research Committee of the University of Hong Kong under Project 10401468.

\section{References}

1. T.-C. Poon, Optical Scanning Holography with MATLAB (Springer, 2007).

2. G. Indebetouw, "Properties of a canning holographic microscope: Improved resolution, extended depth-of-focus, and/or optical sectioning, J. Mod. Opt. 49, 1479-1500 (2002).

3. G. Indebetouw, and W. Zhong, "Scanning holographic microscopy of three-dimensional fluorescent specimens," J. Opt. Soc. Am. A 23, 1699-1707 (2006).

4. T. Kim, "Optical sectioning by optical scanning holography and a Wiener filter," Appl. Opt. 45, 872-879 (2006).

5. H. Kim, S. Min, B. Lee, and T. Poon, "Optical sectioning for optical scanning holography using phase-space filtering with Wigner distribution functions," Appl. Opt. 47, D164-D175 (2008).

6. E. Y. Lam, X. Zhang, H. Vo, T. Poon, and G. Indebetouw, "Three-dimensional microscopy and sectional image reconstruction using optical scanning holography," Appl. Opt. 48, H113-H119 (2009).

7. X. Zhang, and E. Y. Lam, "Edge-preserving sectional image reconstruction in optical scanning holography," J. Opt. Soc. Am. A 27, 1630-1637 (2010).

8. Z. Xin, K. Dobson, Y. Shinoda, and T. Poon, "Sectional image reconstruction in optical scanning holography using a random-phase pupil," Opt. Lett. 35, 2934-2936 (2010).

9. X. Zhang, and E. Y. Lam, "Edge detection of three-dimensional object by manipulating pupil functions in optical scanning holography system," in IEEE International Conference on Image Processing (IEEE, 2010), pp. 3661-3664.

10. J. Ke, P. Shankar, and M. A. Neifeld, "Distributed imaging using an array of compressive cameras," Opt. Commun. 282, 185-197 (2009).

11. J. Ke, A. Ashok, and M. A. Neifeld, "Object reconstruction from adaptive compressive measurements in feature-specific imaging," Appl. Opt. 49, H27-H39 (2010).

12. M. A. Neifeld, and J. Ke, "Optical architectures for compressive imaging," Appl. Opt. 46, 5293-5303 (2007).

13. X. Zhang, E. Y. Lam, and T. Poon, "Reconstruction of sectional images in holography using inverse imaging," Opt. Express 16, 17215-17226 (2008).

14. X. Zhang, and E. Y. Lam, "Sectional image reconstruction in optical scanning holography using compressed sensing, in IEEE International Conference on Image Processing (IEEE, 2010).

15. S. H. Chan, A. K. Wong, and E. Y. Lam, "Initialization for robust inverse synthesis of phase-shifting masks in optical projection lithography," Opt. Express 16, 14746-14760 (2008).

16. Z. Bai, and Z. Wang, "Restrictive preconditioners for conjugate gradient methods for symmetric positive definite linear systems," J. Comput. Appl. Math. 187, 202-226 (2006).

17. X. Zhang, E. Y. Lam, T. Kim, Y. S. Kim, and T.-C. Poon, "Blind sectional image reconstruction for optical scanning holography," Opt. Lett. 34, 3098-3100 (2009).

18. J. W. Goodman, Introduction to Fourier Optics, 3rd ed. (Roberts and Company, 2004).

19. J. Wilson, and J. F. B. Hawkes, Optoelectronics: An Introduction (Prentice Hall, 1993). 